\title{
Subjektivation und ästhetische Bildung in der post-digitalen Kultur
}

\author{
Benjamin Jörissen
}

Friedrich-Alexander Universität Erlangen-Nürnberg, Institut für Pädagogik, Pädagogik, Bismarckstraße 1a, D-91054 Erlangen

benjamin.joerissen@fau.de

\begin{abstract}
The term >Post-digital culture $<$ describes a condition where digitality is deeply embedded not only into the medial aspects of everyday life, but also into the infrastructural and material layers of culture. The conditions of processes of subjectivation are transformed through the digital designs on multiple levels. Aesthetic education is discussed as a particular doorway to relate to transformations of the sensual, medial and material conditions of subjectivation through digital designs.
\end{abstract}

Wenn im Titel dieses Beitrags von >post-digitaler Kultur< die Rede ist, so ist es ratsam, vorab zwei Missverständnisse auszuschließen. Zum einen möge das diagnostische Präfix >post- $<$ nicht etwa in dem Sinne verstanden werden, dass die Phase der Digitalisierung sich nunmehr ihrem Ende zuneigte - im Gegenteil. Zum anderen markiert es durchaus nicht eine Gegenthese zur derzeit verbreiteten These von der >digitalen Kultur< das (vgl. Stalder 2016; Reichert/ Richterich 2015) - soll vielmehr erstens den technikzentristischen Anklang dieser Formel vermeiden (der diesem durchaus nicht technikzentristischen Diskurs nicht gerecht wird), und zweitens Verwechslungen vorbeugen insbesondere mit der seit langem diskutierten und erforschten Kulturalität der sozialen Praktiken >im< Internet, also etwa der Online-Kultur (vgl. Sandbothe/ Marotzki 2000).

Mit >post-digitaler $<$ Kultur ist also nichts anderes als Kultur unter Bedingungen vielfältiger und komplexer digitaler Transformationen und Disruptionen 
gemeint (vgl. Andersen 2014; Berry/Dieter 2015; Jörissen 2016; Stalder 2016), die längst nicht nur auf medialer und kommunikativer Ebene zu verorten sind, sondern auch und vor allem auf den Ebenen der Ökonomie, der Logistik, der Infrastrukturen, der Produktionsmittel, der Organisation von Sozialität, und nicht zuletzt auch im engeren Sinne auf anthropologischer Ebene, bezogen etwa auf technogene Transformationen menschlicher Perfektibilität (vgl. Duttweiler et al. 2016), auf tiefgreifende Verschiebungen im Verhältnis von Mensch, Bild und Imagination (vgl. Cubitt 2014), auf das Entstehen neuer epistemischer Akteure (vgl. Parisi 2013; Hörl/Parisi 2013). ${ }^{1}$

\section{1 >Kultur< und >ästhetische Bildung}

Die Rede von >digitaler oder eben auch >post-digitaler Kultur setzt allerdings auch an einem je zu klärenden Kulturbegriff an. Während >Kultur< im breiten Sinne zeitdiagnostischer Bezeichnungen als Containerbegriff für >das Ganze menschlicher Praxis« stehen kann, werden in diesem Beitrag, in einem engeren Sinne, darunter die (empirisch und historiographisch rekonstruierbaren) Formgefüge verstanden, die aus sozialen Praktiken hervorgehen, in sozialen Praktiken tradiert und transformiert werden (Schäfer 2013) und die ihrerseits Sinnanschlüsse, und somit Sozialität, überhaupt erst ermöglichen (vgl. Hahn 2000). >Kulturく in diesem basalen Verständnis ist ein Phänomen, das sich in kollektiven symbolischen Orientierungs- und Artikulationsformen (Habitus, Gesten-, Bild-, Sprachformen; vgl. Alkemayer/Budde/Freist 2013), in Routinen, Ritualisierungen und Ritualen (Umgangsformen, Alltagsrituale, Feste, Feiern; vgl. Wulf et al. 2001), auch in Formaspekten materieller und infrastruktureller Konfigurationen (Artefakte, Bauten, Trägermedien, Kommunikationssysteme, Technologien, Topologien; vgl. Nohl/Wulf 2013; Göbel/Prinz 2015; Kalthoff/ Cress/Röhl 2016; Weiß 2017; Sexton 2017) sowie in institutionellen und politischen Formen (z.B. in Bezug auf Geschlechterordnung, kollektive Lebensformen wie Ehe, Familie und Clan, generationale Identitäten, Funktionen und Hierarchien; vgl. Geertz 1983) artikuliert. Diese Formgefüge gehen immer wieder - iterativ - als emergentes Moment aus praktischen Vollzügen hervor, innerhalb derer sie als symbolische Anordnungen in Erscheinung treten, die Deutungspraktiken ermöglichen, also Bedeutungen erzeugen und als bedeutsam erscheinen. Kultur ist in dieser Hinsicht als Ineinandergreifen von stabilen und instabilen Prozessqualitäten in der Zeit zu verstehen (vgl. Schäfer 2013). Ihre relative Stabilität ermöglicht überhaupt erst, sie als Form zu beobachten und sie schließlich - durch immer schon enkulturierte Beobachter_innen von Kultur, mithin selbstreferenziell - als Kultur zu bezeichnen. Umgekehrt ist eine relative Instabilität von Kultur hierfür gleichermaßen Voraussetzung, denn erst die Differenz von einer Form zur vorangehenden macht beide als 
Formen unterscheidbar, somit erkennbar und verhandelbar. Daher ist Kultur immer geschichtlich, immer tradierend und immer zugleich transformativ. ${ }^{2}$

Sind Kulturen zumindest implizit selbstreferenziell, in aller Regel jedoch explizit-reflexiv (also auch mittels dezidierter Semantiken der Selbstbezeichnung) auf sich bezogen, so stellt diese selbstreferenzielle Konstruktion von Kultur als identifizierbare Entität eine Fiktion dar, innerhalb derer sich die diskursive Arena vielfältiger normativer Ausdeutungen von >Kultur` (als Hochkultur, Leitkultur, Soziokultur, Popkultur etc.), wie auch reflexiver Bezugnahmen auf die resultierenden kulturellen Konstruktionen von >Kultur<, entfaltet. Mythen, Philosophie, Geschichtsschreibung, seit Beginn der Moderne auch explizit kulturtheoretische Diskursivierungen, zeigen dies auf unterschiedlichen sozialen Wissensebenen auf (vgl. Cassirer 1923).

Während die avancierten theoretischen Diskurse der Moderne und Postmoderne in ihren jeweilig eigenen Sphären stattfinden und zumeist auf diese beschränkt bleiben, sind es jedoch vor allem die Felder ästhetischer Praktiken von rezeptiven und produktiven ästhetischen Alltagspraktiken bis hin zu institutionalisierten und kanonisierten >Künsten ausbildung von Kunst als eigenlogisches Feld menschlicher Praxis (vgl. Luhmann 2007; Bertram 2014) -, innerhalb derer symbolische Formaspekte von Kultur aufgegriffen, inszeniert und somit sinnlicher Erfahrung, ästhetischen und auch propositionalen Urteilen zugänglich werden. Eine solche Praxis ästhetischer Bezugnahme ist dadurch definiert, dass sie Formaspekte von Kultur derart in ein Verhältnis zu sinnlichen Wahrnehmungspraxen setzt, dass diese im Prozess oder seinen Resultaten auf je bestimmte Weise gegenständlich - in diesem Sinne artikuliert - werden. Als Praxis einer solchen artikulativen Relationierung wird schließlich auch >ästhetische Bildung $`$ als grundlegender Modus von Bildungsprozessen verständlich (der im Übrigen auch den ambivalenten, hegemonialen und gouvernementalen Momenten von Bildung [vgl. Ricken 2006] nicht entkommt).

Ästhetische Bildung im Sinne transformativer, sinnlich-artikulativer relationaler Praktiken ist zwar in besonderer Weise auf diejenigen kulturellen Repertoires ästhetischer Artikulationsmöglichkeiten verwiesen, die arbiträr oder qua Systemschließung als $>$ Kunst $<$ je historisch institutionalisiert werden; sie kann jedoch ebenso auf emergenten und nicht oder nur schwach institutionalisierten ästhetischen Artikulationsrepertoires aufsetzen. Daher hat ästhetische Bildung zum einen wenig bis nichts mit hochkulturell fokussierenden Vorstellungen >kultureller Bildung« im Sinne der Hervorbringung >gebildeter<, oder auch nur hinreichend genuss- und konsumfähiger Kulturconaisseure (in einem lediglich feuilletonistischen Sinn von >Kultur<, der in der Kulturpädagogik nichts verloren hätte) zu tun. Zum anderen operiert sie in diesem 
Verständnis jenseits der klassischen Versprechungen ästhetischer Bildung (vgl. Ehrenspeck 2013, S. 87ff), indem sie nicht als erbauliches, moralisierendes Großprojekt, sondern als kritisches Moment in Subjektivationsprozessen begriffen wird. Kritik in diesem Sinne dabei zu verstehen als »Kritik einer institutionalisierten Praxis, eines Diskurses, einer Episteme, einer Institution, und sie verliert ihren Charakter in dem Augenblick, in dem von dieser Tätigkeit abgesehen wird und sie nur noch als rein verallgemeinerbare Praxis dasteht « (Butler 2012). Ästhetische Praxis wird dann insbesondere in ihren transgressiven Potenzialen als Möglichkeit kritischer Praxis bildungsrelevant (vgl. Jörissen 2013).

\section{2 Ästhetische Bildung als relationaler Prozess}

>Artikulative Relationierung< im oben genannten Sinne ist vor diesem Hintergrund als Subjektivationsprozess zu verstehen, insofern das Subjekt nicht nur >etwas< artikuliert, sondern es aus artikulativen Prozessen überhaupt erst hervorgeht. Diese Vorstellung vom Status dessen, was man traditionellerweise als Subjekt zu adressieren und in einer objektiven Sphäre (>Kultur<, >Gesellschaft<) zu verorten gewohnt ist, kann insbesondere dort irritieren, wo Bildung verkürzt als >subjektive Seite der Kultur verstanden und somit die historische und genetische Prozessualität sowohl von Subjektivität als auch von Kultur (Horkheimer/Adorno 1969) ausgeblendet wird. Solche, >Kultur< und >Subjekt< substanzialistisch festschreibenden Auffassungen, setzen durch die Rhetorik der >subjektiven< vs. >objektiven Seite < die autonome Existenz dieser Sphären sprachlich, also auch logisch, voraus. Nichts weist jedoch in den anthropologischen, subjekt- und identitätstheoretischen Diskursen darauf hin, dass so etwas wie ein Subjekt sich als fixierter wissenschaftlicher Gegenstand haltbar konstruieren lässt. Die historische Diversität der (kulturell disponierten) Formen menschlicher Selbstverhältnisse (vgl. etwa Taylor 1991; Zima 200o; Reckwitz 2006; Zirfas/Jörissen 2007) verweist vielmehr darauf, dass - je in sozialen Praxen situiert - >Subjekte< nicht nur (sozialisatorisch und erzieherisch) hervorgebracht werden (vgl. Veith 2001), sondern dass Subjektivität auch stets über Anerkennungs- und Anrufungsprozesse stabilisiert werden muss (vgl. Honneth 1994; Butler 2001).

Die Relationalität des gleichermaßen von Kultur durchzogenen Somatischen und Sozialen, aus der Subjektivität je hervorgeht, diskutiert die Philosophin Juliane Rebentisch in einem philosophisch situierten, bildungstheoretisch ausgesprochen spannenden Blick auf den Zusammenhang von ästhetischer Freiheit und Selbstbestimmung (vgl. Rebentisch 2012). Ihre Relektüre des romantischen Subjektverständnisses begegnet den in der Pädagogik allseits spürbaren, und vielerorts problematisierten Tendenzen neoliberaler Funktionalisierung von Ausdruck, Freiheit, Kreativität, Flexibilität, Tentativität und >Bildung، 
(in bestimmten zugerichteten Verständnissen des Wortes) auf eine Weise, die durchaus eine nähere Betrachtung verdient. ${ }^{3}$ Dabei setzt sie gegen $» d i e$ in der Philosophie dominante Tradition, welche Freiheit einseitig mit dem Vermögen zur vernunftgemäßen Selbstbestimmung gleichsetzt« (Rebentisch 2012, S. 184) - und dies gilt, wie sich ergänzen ließe, durchaus auch für autonomietheoretische Subjekt- und Kompetenzfixierungen in pädagogischen Diskursen »die romantische Integration von unwillkürlichen, irrationalen, anarchischen Impulsen [...], das, was Adorno >Dialektik der Freiheit ' genannt hat: ein im Herzen des Begriffs wirksamer Antagonismus, der das Vermögen zur Selbstbestimmung mit einem ihm gegenläufigen Impuls zusammenspannt« (ebd.).

Diese a-rationale >innere Natur<, die freilich seit der Romantik vielfältige diskursive Karrieren hinter sich gebracht hat (vgl. Gödde 2009; von Braun/ Dornhof/Johach 2009), ist, soviel lässt sich über die heterogenen Versuche ihrer Entschlüsselung als wohlbekannt festhalten, nicht objektivierbar. Wesentlich jedoch ist, dass sie gleichwohl nicht verabsolutiert werden darf: die Verabsolutierung eines schon aus logischen Gründen nicht Objektivierbaren wäre nichts anderes als Metaphysik, die wiederum den subjektivistischen Reflex einer apriorischen Absonderung des Subjekts in eine abermals enthobene Sphäre verlegte. Wenn Rebentisch zu Recht darauf hinweist, dass unsere entzogene >innere Natur nur im Modus des Ereignisses zugänglich ist: nicht als statische Immanenz, sondern »uns zugänglich allein durch das Verhältnis, in dem wir immer schon zu unserer Umwelt stehen « (Rebentisch 2012, S. 181), so ist dies ein maßgeblicher Hinweis darauf, dieses eben nur vermeintlich >Innere< nun schließlich - auch empirisch - dort zu suchen, wo es in Erscheinung tritt: in den situativen Praktiken der Bezogenheit innerhalb der Netzwerke aus Körpern, Dingen, Räumen, Medialitäten, Symbolen, epistemischen Strukturen und Akteuren, kulturellen Formen, materiellen Praktiken und Dispositiven, sinnlichen, ästhetischen, kognitiven Wahrnehmungsereignissen.

An dieser Stelle kann nun einsichtig werden, dass der theoretische Verzicht auf die vorgängige Setzung des Subjekts >Subjektivität $<$ nicht als solche verwirft, sondern im Gegenteil diese als wesentliches Moment - aber eben als Moment, und das heißt: als ein Moment in Beziehung zu anderen Momenten verstehen lässt. Subjektivität im emphatischen Sinn ist selbst nicht Einheit einer Substanz (Identität), sondern im Gegenteil Differenz von Prozess und Ereignis. ${ }^{4}$ Was sich ereignishaft und situativ artikuliert, ist in seiner disruptiven Qualität durchaus eine Kraft: Doch die »Kraft solcher Bestrebungen ist [...] nicht als substanzielle Größe zu verstehen, die der Einheit des Subjekts (oder seinem somatischen Kern) zugerechnet werden könnte. [...] Aufgrund ihres konstellativen Charakters gehört die Kraft solcher Bestrebungen nicht allein dem Subjekt zu. Dennoch wird das Subjekt durch das Ereignis solcher Bestrebungen, die es aus seinem jeweiligen sozialen Selbstverständnis herausrücken, zugleich mit 
einer Dimension seiner selbst konfrontiert, die ihm unverfügbar (vor-) gegeben ist. [...] >Innere Natur $<$ ist in diesem Verständnis indes kein Programm, das sich in Kultur, die Kultur einer sozialen Identität, umschreiben lässt; im Gegenteil: sie macht sich als deren jeweilige Krise bemerkbar« (ebd., S. 18of).

Rebentisch sucht in ihrer Relektüre der romantischen Auffassung ästhetischer Freiheit nach einem Weg, die soziale Konstituiertheit des Subjekts in den Blick nehmen zu können, und dennoch - oder vielmehr: aus diesem Blick heraus - ein Verständnis von Freiheit sichtbar zu machen, das sich deutlich von neoliberal-subjektivistischen Freiheitsverständnissen abgrenzt. Damit empfiehlt sich, bildungstheoretisch gewendet, die Bedeutung des Ästhetischen als eine alternative, wesentlich ereignishafte und somit nicht kontrollierbare Quelle der Relationierung, - einer Relationierung, der Sozialität und Kulturalität zutiefst eingeschrieben sind - denn es >gibt< keine innere Natur jenseits von Kultur und Sozialität; das >Innere $<$ ist als gerichtetes Ausdrucksgeschehen schon Bezogenheit; seine Äußerung ist immer kulturell durchdrungene Artikulation, wenn sie irgendwie als Zeichen anerkennbar sein soll (vgl. Taylor 1992; Jung 2009) - daher steht auch die ästhetische Artikulation keineswegs außerhalb normativer Rahmungen, wenn sie sich auch immer implizit von diesen zu lösen und zu ihnen verhalten vermag: Das Selbst »formt sich immer im Rahmen von Formierungspraktiken, die ihm vorangehen« (Ricken 2006, S. 101).

Vor diesem Hintergrund ist 〉Bildung \ daher nicht mehr plausibel als transformativer Prozess konstruierbar, der lediglich >in< einem Subjekt verortet wird, also auf subjektive Bewusstseins- oder Wissensphänomen beschränkt gedacht wird.

»Die so verstandene Dialektik der Freiheit vollzieht sich [...] nicht jenseits des Sozialen, sondern als dessen Dynamisierung. Tatsächlich verstünde ich die Veränderung meiner selbst falsch, verstünde ich sie als rein private Veränderungen. Durch sie verändere ich die Praxis, deren Teil ich bin. [...] Dass ich mich von nun an anders verstehen will, kann auch heißen, dass ich [...] in einen Kampf um Anerkennung eintreten muss. Die Welt muss dann, damit ich sie weiterhin bewohnen kann, eine andere werden. «

(REBENTISCH 2012, S. 185)

\subsection{Subjektivation unter $>$ post-digitalen $<$ Bedingungen}

Die >vorangehenden Formierungspraktiken $<$ - damit zurück zur These der Post-Digitalität - werden durch transformative kulturelle Prozesse tiefgreifend verändert. Dies kann analytisch im Spannungsfeld von Materialität, Sozialität, Medialität und Kulturalität beobachtet werden, wobei diese vier Momente nicht etwa als autarke Sphäre, sondern als gegenseitig füreinander konstitutive 
Bedingungsmomente zu verstehen wären: keine Kulturalität und keine Sozialtät ohne Symbol, ergo Medialität (vgl. Schwemmer 2005); keine Medialität ohne Materialität (vgl. Mersch 2002), keine Materialität (Dinglichkeit, Körperlichkeit, Räumlichkeit) außerhalb der kulturellen Formen, Dispositive und sozialen Praktiken, durch die sie - je nach Beobachterperspektive - epistemisch (vgl. etwa Foucault 1974, Rorty 1980), phänomenal (vgl. etwa Meyer-Drawe 1984), akteurstheoretisch (vgl. etwa Latour 1993) oder praxeologisch (vgl. etwa Butler 1997; Löw 2001; Hörning/Reuter 2004; Alkemeyer/Budde/Freist 2013) konstituiert werden.

Verschiebungen in den Bedingungsgefügen von Subjektivationsprozessen in unserem Kontext digitalisierungsbedingte Transformationen - können entlang der Heuristik dieser vier Momente thematisiert werden. Dies ist in Bezug auf das bereits an sich ausgesprochen komplexe Phänomen der Digitalität hilfreich, insofern es erlaubt, diese aus dem engeren Fokus medialer Phänomene (v.a. im Sinne einer Einengung von >Digitalisierung< auf >digitale Mediatisierung < etwa im Sinne von Krotz 2001) herauszulösen. Digitalität erscheint dann (etwa) als Spannungsfeld von digitaler Materialität (vgl. Reichert et al. 2015), Software (als spezifisch performanter Medialität, vgl. Chun 2011); digitalen Netzwerklogiken (als spezifische Transformation von Relationalität und Sozialität; vgl. etwa Faßler 2001; Galloway/Thacker 2007), und dem Phänomen der Datenbank - als datenförmige Konstruktion von Welt und insofern als neue kulturelle Form (Manovich 1999; Poster 2001). Ich habe dies in bildungstheoretischer Hinsicht an anderer Stelle in Bezug auf Netzwerke (vgl. Jörissen 2016c), Software (vgl. Jörissen/Verstaendig 2016) und in genealogischer Perspektive auf den Konnex von Daten, Subjektivität und Digitalität diskutiert (vgl. Jörissen 2016a), was aus Raumgründen hier nicht aufgegriffen werden kann. Nachfolgend möchte ich mich daher insbesondere auf die materiellen Aspekte von Digitalität und Digitalisierung beziehen.

Materialität ist (a) Grundlage, aber auch (b) Gegenstand und schließlich zunehmend - (c) Produkt von Digitalität.

(a) In Bezug auf ersteres greift der technologiezentrierte Blick auf $>$ Hardware durchaus zu kurz, und zwar in mehrfacher Hinsicht. Zumeist mit den Bauteilen von Computern identifiziert, suggeriert er eine Substanzialität qua dinglicher Abgeschlossenheit (im Sinne eines Computersystems als Integration von Recheneinheiten, Speichern und Bedienungsinterfaces), die zwar in dieser Form existiert, aber nicht (mehr) eine paradigmatisch zureichende Analysegrundlage von Digitalität darstellen kann, wie es in der früheren 
Medienphilosophie der Fall war (vgl. Bolz/Kittler/Tholen 1994). Nicht nur kulturtheoretisch, sondern auch im Hinblick auf das Moment der stetigen (marktgetriebenen) Evolution von $>$ Hardware $<$ - wie sie schließlich in der >geplanten Obsoleszenz« selbst zum ökonomischen Bewegungsprinzip ihrer Entwicklung wird - ist >Hardware < längst nicht so >hart< wie der Begriff suggeriert; sie ist im Gegenteil ausgesprochen fluide. Hardware ist, wie die immer weiter beschleunigten Entwicklungszyklen der letzten Jahrzehnte im Hinblick auf Leistungsfähigkeit, Miniaturisierung und Mobilisierung verdeutlichen, immer nur momentaner >Ausdruck eines technologisch-kulturellen Fortentwicklungsprozesses von Digitalität.

Die Materialität von Digitalität wird in diesem Sinne als primär transitorisches Moment verstanden, was zugleich bedeutet, sie nicht so sehr im statischen Blick auf Einzelgeräte, sondern in ihren Prozessdynamiken zu verstehen. Ohne an dieser Stelle in Technologieexkurse abgleiten zu wollen (und zu können), können dabei die Miniaturisierung und die damit ermöglichte Komplexitätssteigerung der Architekturen von >Processing Units $<$ (PU, wie in CPU oder GPU) und die Miniaturisierung nicht nur medialer, sondern auch sensorischer und materieller Interfaces als wichtige Phänomene in den Blick geraten. Diese beiden Steigerungsdynamiken tragen ihren Grund nicht >in sich $<$ im Sinne technologisch gegebener Notwendigkeiten (etwa der idealen Prozessorgeschwindigkeit, für die ein Taschenrechner nötig ist). Sie stehen vielmehr in einem Verhältnis zu menschlichen (individuellen, sozialen, ökonomischen etc.) Vollzügen. Wir sehen am Beispiel aktueller >deep-learning«-Anwendungen, wie die Kombination aus Verarbeitungsgeschwindigkeit und -komplexität (zumindest initial) sich am menschlichen Maß bemisst, wenn zum einen die Entwicklung >neuromorpher $\mathrm{PU}$ (wie etwa der TrueNorth-Chip von IвM) nicht mehr der klassischen Von-Neumann-Architektur, sondern einer dem Neocortex nachempfundenen Verschaltungslogik folgt, und wenn $>$ Meilensteine $<$ der Entwicklung >Künstlicher Intelligenzく (KI) sich an Grenzphänomenen kognitiver Komplexität, insbesondere an situativen Spielvollzügen wie bei Schach oder Go, orientieren. Als >initiak bezeichne ich diese Prozesse deshalb, weil sie sich vom Maß des Menschlichen durchaus auch emanzipieren können. ${ }^{5}$

Anthropologisch und damit bildungstheoretisch relevant ist also die relativ zu menschlichen Sinnen und Verarbeitungsgeschwindigkeiten hohe und zunehmend höhere >Feinkörnigkeit $<$ digitaler Interfaces: die Kapazität von Mikroprozessoren zu einer quantitativen und temporalen Auflösung, welche die statische und dynamische Qualität der Ausgabe in Relation zu den spezifisch menschlichen sinnlichen Wahrnehmungskapazitäten bestimmen, die Feinheiten von Interface-Technologien wie den (nicht zufällig so benannten) >Retina-Displays« moderner Gadgets, die auch die hohe räumliche und auch 
farbräumliche Auflösung menschlicher visueller Wahrnehmung übertreffen, lassen informationale Prozesse auf besondere Weise in ein Verhältnis zu den materiellen Bedingungen von Wahrnehmung und Erfahrung treten. Dies wurde zunächst im medienwissenschaftlichen Kontext anhand der Entwicklung digitaler Remediatisierung sichtbar (vgl. Mersch 2002).

(b) Die Digitalisierung analoger Medialität bezog sich zunächst lediglich auf das erscheinende Moment des Mediatisierungsprozesses: Die Digitalisierung eines Textes, der Scan eines Fotos oder das Sampling von Klängen operieren auf der Ebene der sinnlichen Wahrnehmungsweisen - der Lesbarkeit des Textes, Erkennbarkeit des digitalen Fotos, Hörbarkeit des Samples. Der lange Weg zu einer Auflösung, die die menschlichen Sinne übertrifft, setzte im Wesentlichen in den späten 1970er Jahren ein - mit grob auflösenden Scan- und Sampletechnologien, entwickelte sich zunächst wesentlich auf der Ebene der Entwicklung psychosensorischer Datenkompressionsverfahren (mp3, jpg usw.) und hat erst in jüngerer Zeit Schwellen der Sinneswahrnehmung so weit über- und Schwellen der wirtschaftlichen Produktionskosten so weit unterschritten, dass auf dieser Ebene allenfalls graduelle Steigerungen (die zumeist nur noch an den Zahlen der technischen Manuale erkennbar sind) von Bedeutung sind.

Das Signum der anschließenden Phase der Digitalisierung analoger Medialität unter Bedingungen erheblich gesteigerter Rechenkapazitäten und verfeinerter Interfacetechnologien liegt jedoch nicht in der Remediatisierung der erscheinenden medialen Phänomene, sondern vielmehr in der Digitalisierung der ihnen zugrundeliegenden physikalischen Materialität. Im Bereich von auditiven und visuellen Medien bedeutet das etwa, dass nicht die statischen (Klang-)Bilder digital emuliert, sondern die dynamischen physikalischen Eigenschaften der Klang- und Bilderzeuger digital simuliert werden (unter Stichworten wie >physical modelingく, >analog modeling< etc.). Charakteristischerweise tritt hierbei ein inszenatorisches Spiel mit den Kontingenzen und Fehlerstrukturen physikalischer und analoger Medialität auf, die im Sinne einer Inszenierung materieller Authentizität (vgl. Fischer-Lichte 2007), allerdings in sogenannter $>$ Echtzeit $<$, beliebige Fehlercharakteristiken bietet: vom Großbildnegativ über unterschiedliche Kleinbild-Filme bis hin zur Instantfotografie, von der Schellackplatte bis zum 8-Bit-Sampler der 1980er Jahre. Mit hohem Aufwand überführt beispielsweise der Hersteller der Software >Pianoteq historische Tasteninstrumente mitsamt Alterserscheinungen - die aber auch deaktiviert werden können - in virtuell-materielle Modelle. Im Bereich populärer Klangwelten werden Schaltkreise analoger Synthesizer, Charakteristika analoger Verstärker-Röhren (etwa für Gitarrenverstärker), Laufunruhen elektromechanischer Orgeln, übersprechende Tonsignale aufgrund mangelnder Kabelisolierung, verstaubte Tastenkontakte, Schwankungsverhalten von 
Widerständen und Kondensatoren, aber auch die physikalischen Eigenschaften historischer Lautsprechersysteme - und schließlich Mikrophone, mit denen sie >abgenommen< und analog aufgezeichnet wurden, simuliert.

Technologien wie die genannten sind so weit verbreitet und >normalisiert<, dass eine entsprechende Software bisweilen nicht mehr kostet als ein Laib Brot. Die Fokussierung auf die möglichst detailgenaue Simulation von Materialität inklusive ihrer Kontingenzen erzeugt nicht einfache Abbilder keine reinen, idealisierten, >platonischen $<$ Gebilde, wie sie klassischerweise als Computersimulation auftraten -, sondern materiell kontaminierte, begrenzte, teilweise verfallende und in jedem Fall dynamisch responsive (virtuelle) Instrumente, Automaten und Maschinen - zutreffend ist hierfür, was Elena Esposito vor langer Zeit über virtuelle Realität notierte: dass es dabei nicht um falsche reale Objekte, sondern um wahre virtuelle Objekte handle (vgl. Esposito 1998, S. 270).

(c) Dass hier exemplarisch Gegenstände ästhetischer Praktiken angeführt werden - mediale Erzeuger im weitesten Sinn -, ist nicht zuletzt dem Umstand geschuldet, dass es sich hier nicht nur um eine für ästhetisierte und mediatisierte Kultur wesentliche Anwendungssphäre digitaler Technologien handelt. Es ist ebenfalls einem habitualisierten Beobachtungsschema von Digitalität (und seinen Pfadabhängigkeiten) geschuldet, dass diese im Bereich des Medialen und sinnlich Wahrnehmbaren, weniger jedoch im Bereich nicht (primär) medialer materieller Prozesse und Phänomene verortet sind. Dennoch ist dieses dritte Moment, das in paradoxaler Anmutung als digitale Hervorbindung materieller Phänomene bezeichnet werden kann, im Hinblick auf die Bedingungen von Subjektivation in der post-digitalen Kultur mindestens ebenso bedeutsam. Wenn materielle Konstellationen >Produkt< von Digitalität sind, dann ist das zunächst in dem Sinne gemeint, dass zunehmend pseudo-materielle Konstellationen zu verzeichnen sind, die sich jedoch nur als hybride materiell-digitale Konstellationen verstehen lassen. Die umständliche Rede von >Konstellationen< (statt etwa von >Dingen< oder >Artefakten<) soll dabei einer naiven Übertragung tradierter (materieller) Wahrnehmung von Dingen vorbeugen:

Denn erstens unterliegen hybride digital-materielle Artefakte der oben genannten >fluiden $<$ Charakteristik von Hardware, deren materielle Konstellationen (wie Software) bereits auf ihre nächsten Versionen angelegt sind - sie sind insofern ephemere Momente innerhalb übergreifender, langfristig geplanter Design-Prozesse.

Zweitens ist `Software < als Moment digital-materieller Dinge nicht notwendig >in $<$ einem Ding verortet, selbst wenn sie teilweise >auf $<$ diesem Ding ausgeführt werden kann. Ein Beispiel hierfür wäre ein Schwarm von Flugdrohnen, der von einer koordinierenden Software gesteuert wird (an die sich ihrerseits 
Softwares >innerhalb< der Fluggeräte anschließen) - was aber in Bezug auf vernetzte Softwares >innen< und >außen< bedeutet, was also in diesem Fall die >Identität $<$ des hybriden Objekts ist, bleibt unklar. Die Grenzen der Dinglichkeit schwinden damit nicht nur im Sinne der oben genannten Fluidität, sondern auch ganz konkret. Das gilt zumal für die digitale Hybridisierung materieller Räume (vgl. Kitchin/Dodge 2011).

Diese Ambivalenz wird drittens schließlich dort noch überboten, wo materielle Konstellationen eine direkte materielle Artikulation von Software und digitalen Objekten darstellen, also beispielsweise im sogenannten ${ }_{3}$ D-Druck . Zum einen sind die so hergestellten Dinge nicht unbedingt von anderweitig industriell oder handwerklich hergestellten Dingen unterscheidbar - sie mögen lebensweltlich an die geschichtlichen und biographischen Verflechtungen dinglicher Praxis (vgl. Nohl 2011) partizipieren. Zum anderen jedoch sind sie immer nur Exemplar, als imperfekter Ausdruck der sie stets übersteigenden Möglichkeiten einer konkreten Software. Mit dieser Form der automatisierten Produktion von Dingwelten auch außerhalb industrialisierter Produktionswelten werden Artefakte erstens tendenziell von den Bedingungen und Notwendigkeiten handwerklicher Herstellung entkoppelt. Das Bauen innerhalb digitaler Umgebungen und Welten - so wie das >Craften $<$ von Objekten in digitalen Spielumgebungen wie etwa $>$ Minecraft $<$ - wird potenziell zu einem Herstellen auch materieller Dinge, wobei der Einsatz neuer quasi-universeller Werkstoffe - wie druckfähige, feinstgranulare Kunststoffe, Metalle und zementhaltige Baustoffe - eine folgenreiche Verschiebung vom Arbeiten (und Abarbeiten) an der Widerständigkeit der mehr oder weniger stark >eigenstrukturierten< klassischen Materialien (wie Holz, Kunststoff, Metall, Papier) zum Arbeiten und Abarbeiten an den Möglichkeiten von Software, digitalen Objekten und Produktionsmitteln (hier also 3D-Druckern) bedingt. Zweitens aber werden auf diese Weise Dinge herstellbar, die nicht auf non-digitale Weise existieren könnten - einerseits hinsichtlich der Möglichkeiten eines additiven materiellen Druckverfahrens, andererseits aber hinsichtlich der Möglichkeiten der unmittelbaren Einbindung von Software, Algorithmen und (online geteilten) Objekt-Datenarchiven (also einem Direktzugriff auf große Archive digitaler kultureller Objekte). Architektur, Design und Bildende Kunst (insbesondere Plastik) bieten zahlreiche Beispiele für die sich daraus ergebenden Veränderungen (vgl. etwa Vierkant 2010; Parisi 2013; Allahiary/Rourke 2017; siehe auch Jörissen 2016b). Was sich im Bereich der Künste vergleichsweise harmlos ausnimmt, erhält in den sich gegenwärtig abzeichnenden biotechnologischen Anwendungen von materiellen Produktionsverfahren - vom digital automatisierten Biohacking bis zum Ausdruck von Organen (vgl. Thacker 2005; Cho et al. 2015; Vargo 2017) - eine zukünftig anthropologisch erheblich gewichtigere Bedeutung. 
Diese - nie nur technologisch in einem akademisch-abstrakten Sinne, sondern als Technologiedesign in konkreter ökonomischer Absicht zustande kommende - Entwicklung digitaler Materialitäten folgt eigendynamischen Logiken, die auf kulturellen Praxen und tradierten Formbeständen transformativ aufbauen können, diese aber auch disruptiv verändern können. Im Hinblick auf die im letzten Abschnitt angeführten Aspekte (a) der Beziehung zwischen Hardware/ Interfaces, Sinnlichkeit und Wahrnehmung, (b) der digitalen Remediatisierung von Materialität und schließlich (c) der digitalen Hervorbringungen von Materialität wird deutlich, dass die bildungstheoretisch anzuschließenden Fragen im Schnittfeld von Anthropologie, Design und Software verortbar sind. Dies kann hier abschließend (aus Raumgründen leider nur kursorisch) diskutiert werden.

(a) Die Rekonfiguration von Weltverhältnissen durch Digitalisierung beschreibt die Philosophin Luciana Parisi als das kulturhistorisch emergente Auftreten eines neuen Akteurs: »als erfassende Wirklichkeiten bieten Algorithmen [...] einen Begriff maschineller, numerischer Ästhetik [...] und der affektiven Erfahrung. [...] Digitales Design bedarf daher keiner einfachen Neubewertung, sondern es muss als Kernaspekt einer allgemeinen Reformalisierung von Macht begriffen werden, die auf einer Softwareisierung [softwarization] von Logistik, Kommunikation, Management und damit den erweiterten Infrastrukturen der Macht basiert, wie sie in der alltäglichen raum-zeitlichen Erfahrung am Werk sind « (Parisi zit. nach Hörl/ Parisi 2013, S. 48 f). Die durch das menschliche Maß gesetzten Formen von Wahrnehmung (Kognition, Aisthesis) werden zum einen durch digitale Logiken aufgegriffen und umgestaltet - wie am Beispiel der mit den Sinnen konkurrierenden und diese schließlich zumindest teilweise geradezu ausspielenden Interfacetechnologien deutlich wird. Dabei spielen auch und insbesondere Fragen der mit dem Interface-Design gegebenen Machtposition eine maßgebliche Rolle (vgl. auch Galloway 2012). Wahrnehmung und Kognition verschieben sich damit einerseits anthropologisch und andererseits, unvermeidbar, auf der Ebene kultureller Dispositionen und Formen.

(b) Remediatisierungen von Medialitäten und Materialitäten verweisen, beginnend mit Walter Benjamins berühmten Kunstwerkaufsatz (Benjamin 2013), auf kulturelle Verwerfungen. Medientheoretisch scheint Benjamins Diagnose des Verlusts der Aura in der (digitalisierungskritischen) Diagnose einer doppelten Verbergungsstrategie digitaler Medialität - zum einen im Hinblick auf 
die Invisibilisierung der medialen Bedingungen von medialen Erscheinungsprozessen, tieferliegend dann noch einmal im Hinblick auf die Invisibilisierung der digitalen Supposition ihrer zugrundeliegenden Materialitäten. In der Tat bedeutet die digitale Remediatisierung von materiellen Dingen freilich eine Verschiebung vom, wie man in Anlehnung an Wiesing (2005) sagen kann, Reich der Genesis und des Vergänglichen in das der Geltung, des Überzeitlichen. Folgenreich an dieser Verschiebung ist jedoch nicht unbedingt primär dieser Entzug (der im Kontext von Software andererseits durch ihre faktische zeitliche Begrenztheit und Fluidität der Versionswechsel ein Stück weit kompensiert wird; ältere schreibende PC-Nutzer_innen leben mit den Versionsgeschichten ihrer Textverarbeitungen), sondern die rigiden Eigenschaften von Software, die wie ein platonisches Gesetz (vgl. Chun 2001) zugleich Vorschrift und (performativer) Vollzug sind. Materialität kann umgebildet, Software jedoch nur >gehackt< werden. Damit rückt bildungstheoretisch die Logik des Hackens - nicht nur als Software-Hacking, sondern auch als Cultural Hacking (vgl. Düllo/Liebl 2004) im Sinne taktisch motivierter alternativer Relationierungspraktiken in den Blickpunkt.

(c) Die digitale Hervorbringung von materiellen Dingen, Räumen und Körperlichkeiten kann in ihren kulturellen Transformationspotenzialen, so weitgehend diese bereits erscheinen mögen, erst in ersten Ansätzen beobachtet und eingeschätzt werden (vgl. Engel/Jörissen 2018). Insofern Subjektivation als wesentlich über Inkorporierung verlaufender Prozess - maßgeblich auch mit dinglichen und räumlichen Konstellationen und Praktiken verbunden ist, sind hier sehr unterschiedliche Dynamiken zu verzeichnen - einerseits Prozesse, die im Sinne hegemonialer Festschreibungen von Kontrolle und Panoptismen operieren, andererseits solche, die als digital basierte Designs die Grenzen von Dingen und Räumen auflösen und darüber auch bestimmte Positionen von $>$ Nutzer-Subjekten $<$ festschreiben (insofern sich die Zugriffe auf dingliche und materielle Konstellationen damit partizipatorischen Begehren eher entziehen). Bildungstheoretisch deutet dies zunächst darauf, dass materielle Aspekte von Subjektivation unter Bedingungen von Digitalisierung zunehmend dem (theoretisch oder praktisch) reflektierenden Zugriff entzogen sein können - durchaus im Sinne interessierter hegemonialer Besetzungen, die aufgrund ihrer enormen technologischen Komplexität nur schwierig zu hinterfragen sind. Jedoch verweist dies andererseits auf die Notwendigkeit, Bildung unter digitalen Bedingungen verstärkt in ihrer partizipatorischen Dimension zu verstehen. In diesem Sinne verweist der gegenwärtige Diskurs auf die Frage nach der Möglichkeit partizipatorischen Designs (vgl. Mareis/Held/ Joost 2013). Ein solcher, anstelle von Finalität die prozesshafte Überlagerung 
differenter Wissens- und Praxisperspektiven setzender Ansatz, lässt Design als Frage nach dem »Design des Designs« (Ehn 2013, S. 91), bzw. des »Design[s] für Design nach dem Design «(ebd.), reflexiv werden. Die performative Macht des Designs wird dabei nicht negiert, sondern sie wird als Moment der Interaktion organisationaler, menschlicher und dinglicher Akteursgeflechte in diskursiven Praktiken initiiert, die Ehn in Anlehnung an Wittgenstein als »Design-Spiele« (ebd., S. 88) konzipiert. Auf der anderen Linie geht es um ein (zumindest diskurspolitisch reklamiertes) Design, das Partizipation nach dem Modell widerstreitender »Design-Spiele« konzipiert.

Vor dem Hintergrund relationaler Bildungstheorie wird in diesem Sinne deutlich, dass die Frage von Relationierungsweisen und -taktiken hierbei maßgeblich ist, zumal solche Rekonfigurationen, wie auch immer in hegemonialer oder profitorientierter Absicht erschaffen, aufgrund ihrer disruptiven Momente unerwartete Freiräume eröffnen. Die zentrale Aufforderung im bildungstheoretischen Sinn besteht daher in der Öffnung und Erschließung nicht-affirmativer Zugänge und Praktiken. Wenn jedoch in pädagogischen Diskursen, so etwa in der Medienpädagogik, häufig umstandslos vom >Nutzer< (idealisiert zum >kompetenten Nutzer<) die Rede ist, so maskiert dies auf durchaus naive Weise dieses Moment von Subjektivation und affirmiert dadurch letztlich marktaffirmatives und insofern zumindest ein an hegemoniale Logiken anschlussfähiges Modell des Nutzer-Subjekts, anstatt es zum (kognitiven, ästhetischen oder praktischen) Gegenstand positionierender, und das heißt: nicht-affirmativer Relationierungspraktiken zu machen.

\section{Literatur}

Alkemeyer, Thomas/Budde, Gunilla/Freist, Dagmar (2013): Selbst-Bildungen. Soziale und kulturelle Praktiken der Subjektivierung. Bielefeld: transcript.

Benjamin, Walter (2013): Das Kunstwerk im Zeitalter seiner technischen Reproduzierbarkeit [1935]. Frankfurt/Main: Suhrkamp.

Berry, David M./Dieter, Michael (Hrsg.) (2015): Postdigital Aesthetics. Art, Computation And Design. U K: Palgrave Macmillan.

Bertram, Georg W. (2014): Kunst als menschliche Praxis. Eine Ästhetik. Frankfurt/ Main: Suhrkamp.

Bolz, Norbert/Kittler, Friedrich/Tholen, Georg C. (Hrsg.) (1994): Computer als Medium. München: Fink.

Butler, Judith (1997): Körper von Gewicht: die diskursiven Grenzen des Geschlechts. Frankfurt/Main: Suhrkamp.

Butler, Judith (2001): Psyche der Macht. Das Subjekt der Unterwerfung. Frankfurt/ Main: Suhrkamp. 
Cassirer, Ernst (1923): Philosophie der symbolischen Formen. Berlin: Bruno Cassirer. Cho, Dong-Woo/Lee, Jung-Seob/Pati, Falguni/Jung, Jin Woo/Jang, Jinah/Park, Jeong Hun (2015): Organ Printing. San Rafael, CA: Morgan \& Claypool Publishers.

Chun, Wendy H.K. (2011): Programmed Visions. Software and Memory. Cambridge, MA: Mit Press.

Cubitt, Sean (2014): The Practice of Light. A Genealogy of Visual Technologies from Prints to Pixels. Cambridge, MA: MIT Press.

Düllo, Thomas/Liebl, Franz (Hrsg.) (2004): Cultural Hacking: Kunst des Strategischen Handelns (1. Aufl.). Wien, New York: Springer.

Duttweiler, Stefanie/Gugutzer, Robert/Passoth, Jan-Hendrick/Strübing, Jörg (2016): Leben nach Zahlen, Self-Tracking als Optimierungsprojekt? (1. Aufl.). Bielefeld: transcript.

Ehn, Pelle (2013). Partizipation an Dingen des Designs. In: Claudia Mareis/Matthias Held/Gesche Joost (Hrsg.): Wer gestaltet die Gestaltung? Praxis, Theorie und Geschichte des partizipatorischen Designs. Bielefeld: transcript, S. 79-104.

Ehrenspeck, Yvonne (2013): Ästhetik und Bildung. In: Ingrid Gogolin/Harm Kuper/ Heinz-Hermann Krüger/Jürgen Baumert (Hrsg.): Stichwort: Zeitschrift für Erziehungswissenschaft. Wiesbaden: Springer Fachmedien, S. 77-96.

Engel, Juliane/Jörissen, Benjamin (2018): Unsichtbare Sichtbarkeiten. Kontrollverlust und Kontrollphantasmen in öffentlichen und jugendkulturellen Digitalisierungsdiagnosen. In: Thomas Alkemeyer/Nikolaus Buschmann/Thomas Etzemüller (Hrsg.): Gegenwartsdiagnosen. Kulturelle Formen gesellschaftlicher Selbstproblematisierung in der Moderne. Bielefeld: transcript. [im Druck]

Esposito, Elena (1998): Fiktion und Virtualität. In: Sybille Krämer (Hrsg.): Medien Computer - Realität. Wirklichkeitsvorstellungen und Neue Medien. Frankfurt/ Main: Suhrkamp, S. 269-296.

Fischer-Lichte, Erika (Hrsg.) (2007): Inszenierung von Authentizität. Tübingen: A. Francke.

Friebertshäuser, Barbara/Rieger-Ladich, Markus/Wigger, Lothar (2009): Reflexive Erziehungswissenschaft: Forschungsperspektiven im Anschluss an Pierre Bourdieu. Wiesbaden: Springer.

Foucault, Michel (1974): Die Ordnung der Dinge: eine Archäologie der Humanwissenschaften. Frankfurt/Main: Suhrkamp.

Galloway, Alexander R. (2012): The Interface Effect. Cambridge, U K; Malden, MA: John Wiley \& Sons.

Galloway, Alexander R./Thacker, Eugene (2007): The Exploit: A Theory of Networks. Minneapolis, London: University of Minnesota Press.

Gebauer, Gunter/Wulf, Christoph (1998): Spiel - Ritual - Geste: mimetisches Handeln in der sozialen Welt. Reinbek: Rowohlt.

Geertz, Clifford (1983): Dichte Beschreibung: Beiträge zum Verstehen kultureller Systeme. Frankfurt/Main: Suhrkamp. 
Göbel, Hanna K./Prinz, Sophia (2015): Die Sinnlichkeit des Sozialen: Wahrnehmung und materielle Kultur. Bielefeld: transript.

Gödde, Günter (2009):Traditionslinien des »Unbewußten«: Schopenhauer - Nietzsche - Freud. Gießen: Psychosozial-Verlag.

Hahn, Alois (200o): Konstruktionen des Selbst der Welt und der Geschichte. Aufsätze zur Kultursoziologie. Frankfurt/Main: Suhrkamp.

Hegel, Georg W.F. (1970): Vorlesungen über die Ästhetik I. Werke 13. Frankfurt/Main: Suhrkamp.

Hepp, Andreas/Krotz, Friedrich/Moores, Shaun/Winter, Carsten (2006): Konnektivität, Netzwerk und Fluss. In: Andreas Hepp/Friedrich Krotz/Shaun Moores/Carsten Winter (Hrsg.): Konnektivität, Netzwerk und Fluss. Wiesbaden: vs Verlag für Sozialwissenschaften, S. 7-19.

Honneth, Axel (1994): Desintegration: Bruchstücke einer soziologischen Zeitdiagnose. Frankfurt/Main: Fischer. [= Honneth 1994a]

Honneth, Axel (1994): Kampf um Anerkennung: zur moralischen Grammatik sozialer Konflikte. Frankfurt/Main: Suhrkamp. [= Honneth 1994b]

Horkheimer, Max/Adorno, Theodor W. (1969): Dialektik der Aufklärung: philosophische Fragmente [1947]. Frankfurt/Main: Fischer.

Hörl, Erich/Parisi, Luciana (2013): Was heißt Medienästhetik? Zeitschrift für Medienwissenschaft 1/2013, S. 35-51.

Hörning, Karl H./Reuter, Julia (Hrsg.) (2004): Doing Culture: Neue Positionen zum Verhältnis von Kultur und sozialer Praxis (1. Aufl.). Bielefeld: transcript.

Jörissen, Benjamin (2013): Transgressive Artikulationen. Eine Skizze zum Zusammenhang von Kunst, Medialität und Kultureller Bildung. In: Sara Burkhardt/Torsten Meyer/Mario Urlaß (Hrsg.): convention (Bd. 3). München: kopaed, S. 97-102.

Jörissen, Benjamin (2016): >Digitale< Bildung und die Genealogie digitaler Kultur: historiographische Skizzen. MedienPädagogik 25/2016, S. 26-40. [= Jörissen 2016a]

Jörissen, Benjamin (2016): Hegemoniale Ästhetiken und ästhetische Gegenstrategien - Kulturelle Bildung in der postdigitalen Kultur. infodienst 120/2016, S. 13-15. [= Jörissen 2016b]

Jörissen, Benjamin (2016): Zur bildungstheoretischen Relevanz netzwerktheoretischer Diskurse. In: Dan Verständig/Jens Holze/Ralf Biermann (Hrsg.): Von der Bildung zur Medienbildung (Bd. 31). Wiesbaden: vs Verlag für Sozialwissenschaften, S. 231-255. [= Jörissen 2016c]

Jörissen, Benjamin/Verständig, Dan (2016): Code, Software und Subjekt. Zur Relevanz der Critical Software Studies für ein nicht-reduktionistisches Verständnis »digitaler Bildung«. In: Ralf Biermann/Dan Verständig (Hrsg.): Das umkämpfte Netz. Machtund medienbildungstheoretische Analysen zum Digitalen. Wiesbaden: vs Verlag für Sozialwissenschaften, S. 37-50. 
Jörissen, Benjamin/Klepacki, Leopold/Unterberg, Lisa/Engel, Juliane/Flasche, Viktoria/Klepacki, Tanja (2018): Spectra of Transformation. Münster: Waxmann.

Jung, Matthias (2009): Der bewusste Ausdruck. Anthropologie der Artikulation. Berlin: Walter de Gruyter.

Kalthoff, Herbert/Cress, Torsten/Röhl, Tobias (2016): Materialität: Herausforderungen für die Sozial- und Kulturwissenschaften. Paderborn: Fink.

Kitchin, Rob/Dodge, Martin (2011): Code/space: Software and Everyday Life. Cambridge, MA: Mit Press.

Krotz, Friedrich (2001): Die Mediatisierung kommunikativen Handelns. Wiesbaden:vs Verlag für Sozialwissenschaften.

Latour, Bruno (1993): We Have Never Been Modern. Cambridge, MA: Harvard University Press.

Löw, Martina (2001): Raumsoziologie. Frankfurt/Main: Suhrkamp.

Luhmann, Niklas (1997): Die Kunst der Gesellschaft. Frankfurt/Main: Suhrkamp.

Manovich, Lev (1999): Database as Symbolic Form. In: Convergence: The International Journal of Research into New Media Technologies 2/1999, S. 80-99.

Mareis, Claudia/Held, Matthias/Joost, Gesche (Hrsg.) (2013): Wer gestaltet die Gestaltung? Praxis, Theorie und Geschichte des partizipatorischen Designs. Bielefeld: transcript.

Mersch, Dieter (2002): Ereignis und Aura: Untersuchungen zu einer Ästhetik des Performativen. Frankfurt/Main: Suhrkamp.

Meyer, Torsten (2013): Next Art Education (Bd. 29: Kunstpädagogische Positionen). Hrsg. von Andrea Sabisch/Torsten Meyer/Eva Sturm. Hamburg: Univ. Hamburg.

Meyer-Drawe, Käte (1984): Leiblichkeit und Sozialität:phänomenologische Beiträge zu einer pädagogischen Theorie der Inter-Subjektivität. München: Fink.

Nohl, Arnd-Michael (2011): Pädagogik der Dinge. Bad Heilbrunn: Klinkhardt.

Nohl, Arnd-Michael/Wulf, Christoph (2013): Mensch und Ding. Die Materialität pädagogischer Prozesse. Wiesbaden: vs Verlag für Sozialwissenschaften.

Parisi, Luciana (2013): Contagious Architecture: Computation, Aesthetics, and Space. Cambridge, MA: MIT Press.

Rebentisch, Juliane (2012): Hegels Missverständnis der ästhetischen Freiheit. In: Christoph Menke/Juliane Rebentisch (Hrsg.): Kreation und Depression. Freiheit im gegenwärtigen Kapitalismus. Berlin: Kadmos, S. 172-190.

Reckwitz, Andreas (2006): Das hybride Subjekt: eine Theorie der Subjektkulturen von der bürgerlichen Moderne zur Postmoderne. Weilerswist-Metternich: Velbrück.

Reichert, Ramòn/Richterich, Annika/Abend, Pablo/Wenz, Karin/Fuchs, Mathias (Hrsg.) (2015): Digital Culture \& Society. Digital Material/ism. Bielefeld: transcript.

Ricken, Norbert (2006): Die Ordnung der Bildung. Beiträge zu einer Genealogie der Bildung. Wiesbaden: vs Verlag für Sozialwissenschaften. 
Rorty, Richard (1980): Philosophy and the Mirror of Nature. Princeton, NJ: Princeton University Press.

Sandbothe, Mike/Marotzki, Winfried (Hrsg.) (2000): Subjektivität und Öffentlichkeit. Kulturwissenschaftliche Grundlagenprobleme virtueller Welten. Köln: Herbert von Halem.

Schäfer, Hilmar (2013): Die Instabilität der Praxis: Reproduktion und Trasnformation des Sozialen in der Praxistheorie. Weilerswist: Velbrück Wissenschaft.

Schwemmer, Oswald (2005): Kulturphilosophie: eine medientheoretische Grundlegung. München: Fink.

Sexton, Kim (2017): Architecture and the Body, Science and Culture. London: Routledge.

Stalder, Felix (2016): Kultur der Digitalität (Originalausgabe). Berlin: Suhrkamp.

Taylor, Charles (1996): Quellen des Selbst. Die Entstehung der neuzeitlichen Identität. Frankfurt/Main: Suhrkamp.

Thacker, Eugene (2006): The Global Genome. Biotechnology, Politics, and Culture. Cambridge, MA: MIT Press.

Trabant, Jürgen (1998). Artikulationen: historische Anthropologie der Sprache. Frankfurt/Main: Suhrkamp.

Tungstall, Elizabeth (2013): Decolonizing design innovation: design anthropology and indigenous knowledge. In: Wendy Gunn/Ton Otto/Rachel C. Smith (Eds.): Design Anthropology. Theory and Practice. London: A\&C Black, p. 232-250.

Vargo, Marc E. (2017): The Weaponizing of Biology. Bioterrorism, Biocrime and Biohacking. Jefferson, NC: McFarland.

Veith, Hermann (2001): Das Selbstverständnis des modernen Menschen. Theorien des vergesellschafteten Individuums im 20. Jahrhundert (1. Aufl.). Frankfurt/Main, New York: Campus.

von Braun, Christina/Dornhof, Dorothea/Johach, Eva (2015): Das Unbewusste. Krisis und Kapital der Wissenschaften. Studien zum Verhältnis von Wissen und Geschlecht. Bielefeld: transcript.

Weiß, Gabriele (2017): Kulturelle Bildung - Bildende Kultur: Schnittmengen von Bildung, Architektur und Kunst. Bielefeld: transcritp.

Wiesing, Lambert (2005): Artifizielle Präsenz. Studien zur Philosophie des Bildes. Frankfurt/Main: Suhrkamp.

Wulf, Christoph/Althans, Birgit/Audehm, Kathrin/Bausch, Constanze/Göhlich, Michael/Sting, Stephan/Tervooren, Anja/Wagner-Willi, Monika/Zirfas, Jörg (2001): Das Soziale als Ritual: Zur performativen Bildung von Gemeinschaften. Wiesbaden: VS Verlag.

Zima, Peter V. (2000): Theorie des Subjekts. Subjektivität und Identität zwischen Moderne und Postmoderne. Stuttgart: чтв GmbH.

Zirfas, Jörg/Jörissen, Benjamin (2007): Phänomenologien der Identität. Human-, sozial- und kulturwissenschaftliche Analysen. Wiesbaden: vs Verlag für Sozialwissenschaften. 


\section{Internetquellen}

Allahyari, Morehshin/Rourke, Daniel (Eds.) (2017): The 3D Additivist Cookbook \& Archive. URL: http://archive.org/details/The3DAdditivistCookbook2o17 (Datum des letzten Abrufs: 17. Januar 2018).

Andersen, Christian U./Cox, Geoff/Papadopoulos, Georgios (Eds.) (2014): POSTDIGITAL RESEARCH | a peer-reviewed journal about_(VOLUME 3, ISSUE 1, 2014). URL: http://www.aprja.net/?page_id=1291 (Datum des letzten Abrufs: 17. Januar 2018).

Cramer, Florian (2015): What is >Post-digital $<$ ? a peer-reviewed journal about. URL: http://www.aprja.net/?p=1318 (Datum des letzten Abrufs: 17. Januar 2018).

Knight, Will (2017): AlphaGo Zero Shows Machines Can Become Superhuman Without Any Help. An upgranded version of the game-playing Al teachesitself every trick in the Go book, using a new form of machine learning. UR L: https://www.technologyreview .com/s/6og141/alphago-zero-shows-machines-can-become-superhuman-without -any-help/ (Datum des letzten Abrufs: 14. Januar 2018).

Vierkant, Artie (2010): The Image Object Post-Internet Artie Vierkant. URL: http:// jstchillin.org/artie/vierkant.html (Datum des letzten Abrufs: 15. Juli 2016).

\section{Endnoten}

1 >Post-Digitalität ist der passende Begriff für einen Zustand, in dem die Digitalisierung so weit fortgeschritten ist, dass das Digitale eine omnipräsente, ubiquitäre Infrastruktur darstellt (vgl. Cramer 2015). Man kann sich dabei das Digitale wie ein Myzel vorstellen: Der eigentliche Organismus besteht aus den unsichtbaren, miteinander zusammenhängenden großen unterirdischen Verflechtungen. Was wir gemeinhin als $>\mathrm{Pilz}<$ bezeichnen, ist lediglich ein Fruchtkörper des Myzels; eine sekundäre Manifestation. Das Digitale ist einerseits ein Netzwerk aus den Maschinen, Leitungen, Satelliten, Software, Algorithmen, Protokollen, Datenstrukturen, Daten, Interfaces, RFID-Sendern, GPS-Sendern, zahllosen Endgeräten mit ihren medialen und sensorischen Komponenten usw. Es ist jedoch mehr, indem die Strukturen digitaler Infrastruktur sich in die materiell-ökonomischen, die kommunikativ-sozialen und die artikulativen und individuellen Sphären, letztlich in die Kultur in ihrer ganzen Breite und Tiefe, längst eingeschrieben haben.

2 >Bildung< lässt sich allein schon deshalb nicht auf statische Konstruktionen von Kultur reduzieren, sondern muss als (oftmals konstitutives) Moment immer als historisch fluider kultureller Transformationsprozesse verstanden werden. 
3 Ihre Argumentationsfolie bezieht sich auf den Rahmen einer freiheitstheoretischen Kritik der - seit Hegel (Hegel 1970) - verkürzten Rezeption romantischer Subjekttheorie, welche den romantischen Diskurs auf die >innere Natur` auf rückzügliche Innerlichkeit, ironische Weltleugnung und vergöttlichtem Geniekult - und somit subjektivistische Ideologie reduziert. Dagegen zeigt Rebentisch anhand der romantischen Praxis der Selbstironie - als exemplarisches Moment - auf, dass im Gegenteil ein faktischer Bezug dieses >Innere< auf die »geltenden sittlichen Gehalte« und die reale »Welt, in der der Ironiker lebt« (Heide 2000 zit. nach Rebentisch 2012, S. 178) notwendig mit einbezogen sind. Die romantische Ironie ist also eine Praxis der Distanzierung, die gleichwohl nur »als ein Moment in der sozialen Praxis« (Rebentisch 2012, S. 179) verstanden werden kann. Was die Romantik praktiziert, ist somit »Ausdruck eines distanzierten Verhältnisses zu den Selbstbildern der zweiten Natur - der eigenen Tugendhaftigkeit ebenso wie der eigenen Genialität. Von deren Bann befreit sich das selbstironische Subjekt offenkundig nicht durch die Behauptung einer diesen noch überlegenen Position eines Gottes, sondern durch deren Brechung in den Grimassen seiner unwillkürlichen Vitalität. In deren Erscheinungen wird sich das Subjekt (und zwar gerade das narzisstisch auf ein überzogenes Selbstbild fixierte Subjekt) selbst fremd - und (über sich lachend) potentiell zu neuen, unter Umständen angemesseneren Bildern seiner selbst frei« (ebd., S. 184).

4 Je nach Perspektivierung kann man allerdings die Einheit dieser Differenz selbst entweder als Prozess reflektieren, d.h. als das Prozessieren ereignishafter Differenzen, wie es in den insofern klassischen Subjektkonstruktionen der Biographieforschung im Vordergrund steht (der transformative biographische Prozess besteht wesentlich aus der sinnhaften Konstruktion, De- und Rekonstruktion von zumeist krisenhaften Ereignissen), oder aber man kann es als Ereignis reflektieren, im Sinne einer Einkerbung auf relationaler Ebene, als lebensgeschichtliche transgressive Diskontinuierung, die ihrerseits Prozesse ermöglichen und somit Artikulationen evozieren, aus denen potenzielle Identitäten hervorgehen.

5 Die zweite Generation der »AlphaGo«-KI der Firma Google, die im Jahr 2016 einen menschlichen Go-Meister besiegt hat, wurde bereits ein Jahr später von der KI »AlphaGo Zero« überzeugend geschlagen im Gegensatz zur ersteren verzichteten die Ingenieure von AlphaGO Zero vollständig darauf, menschliche Spieldaten zum Training der Software zu verwenden (vgl. Knight 2017). Ab einem gewissen Punkt sind kulturhistorisch entstandene, menschliche kognitive Muster offenbar eher hemmend als förderlich im Hinblick auf die Effizienz digitaler Informationsverarbeitung. 